\title{
The influence of a light pipe on the coherence properties in laser projectors
}

\author{
Stijn Roelandt ${ }^{a}$, Jani Tervo $^{b}$, Youri Meuret ${ }^{c}$, Guy Verschaffelt ${ }^{d}$ and Hugo Thienpont ${ }^{a}$ \\ ${ }^{a}$ Brussels Photonics Team B-PHOT, Vrije Universiteit Brussel, \\ Pleinlaan 2, B-1050 Brussels, Belgium; \\ ${ }^{b}$ Institute of Photonics, University of Eastern Finland, \\ P.O. Box 111, FI-80101 Joensuu, Finland; \\ ${ }^{c}$ Light \& Lighting Laboratory, KU Leuven - Campus Gent, \\ Gebroeders De Smetstraat 1, B-9000 Gent, Belgium; \\ ${ }^{d}$ Applied Physics Research Group APHY, Vrije Universiteit Brussel, Pleinlaan 2, B-1050 \\ Brussels, Belgium.
}

\begin{abstract}
Light pipes are key optical components used in projection systems to transport and homogenize light from the source towards the light valve. They can provide a uniform light distribution at their output as a result of multiple internal reflections. In laser projection systems, such light pipes are useful in combination with a laser-light module consisting of one or more single-mode lasers and a rotating diffuser. The partially coherent light emanating from the rotating diffuser is transported and homogenized towards the end of the light pipe. Consequently, propagation through the light pipe will also modify the coherence properties of the laser light. In this paper, a computationally efficient simulation model is presented to propagate partially coherent light through a homogenizing rectangular light pipe. The resulting coherence function clearly differs from that of free-space propagation over the same optical path length. The implications of these results on, for example, the appearance of speckle are discussed in further detail. The simulation results are experimentally verified using a reversing wavefront Michelson interferometer. The approach described in this paper can be extended further to investigate other types of light pipes, such as tapered light pipes or even more complex ones.
\end{abstract}

Keywords: Partial coherence, Speckle, Projection, Illumination system

\section{INTRODUCTION}

In projection applications, laser light sources are gaining attraction due to the possibility of obtaining a wide color gamut and high luminance. ${ }^{1-3}$ Inherently connected to the use of lasers is the appearance of speckle. A speckle pattern is created by interference of (at least partially) coherent radiation that is scattered from a random surface that is rough on the scale of the optical wavelength. ${ }^{4,5}$

The rotation of a diffuser in a laser light beam is an effective method for reducing speckle. ${ }^{6}$ This moving diffuser reduces the coherence area of the laser beam, resulting in a beam of partially coherent light. Propagation of partially coherent light involves, in general, the evaluation of four-dimensional integrals, which is a tremendous computational task. ${ }^{7}$ The computational complexity of propagating such radiation can be significantly reduced if one can represent the partially coherent field in terms of fully coherent, but mutually uncorrelated modes. Several alternative principles for such a decomposition exist, but in the context of this work the so-called shifted elementary-mode approach ${ }^{8-10}$ is probably the most meaningful one. In that approach, the partially coherent field is expressed as a superposition of mutually independent coherent elementary fields, which are identical but originate from different positions at the source plane. The shape of each elementary mode is fully defined by the Fourier transform of the source's far-field intensity pattern. Each mode has the same shape and hence the modes

Further author information: (Send correspondence to Stijn Roelandt) Stijn Roelandt: E-mail: stijn.roelandt@b-phot.org, Telephone: +32 26293658 
create identically-shaped far-field intensity patterns. The far-field intensity pattern after the rotating diffuser has a Gaussian shape. Hence, the elementary modes also need to have a Gaussian shape.

In projection applications, several optical components have the sole purpose of homogenizing the light beam, such as for example a light pipe. This component exerts a large influence on the propagated light. In this paper, we investigate the effect of a light pipe on the coherence properties of light. ${ }^{11}$ We will do this both experimentally and based on numerical simulations, where the experiments confirm the validity of the numerical model while the modelling provides a better insight into the influence of the various setup parameters.

Propagation of partially coherent light can be investigated on the basis of field tracing ${ }^{12}$ and on a ray-tracing basis. ${ }^{13}$ However, interaction of partially coherent light with optical structures that perform complex optical tasks, such as homogenization, either become too cumbersome to simulate on a field tracing approach or are oversimplified in ray-tracing, i.e. many diffraction effects are neglected. The simulation model described in this paper is capable of investigating these situations and the results are experimentally confirmed.

In Section 2, the investigated configuration is elaborated on and the simulation model is described together with the representation of the source, the behavior of a light pipe and the evaluation of the coherence properties. The experimental verification method is discussed in Section 3. The interaction of the light with the light pipe is described in Section 4. Section 5 elaborates on the consequences and outlook, and provides a conclusion of the investigation.

\section{OPTICAL SIMULATION MODEL}

The illumination setup investigated in the following paper is illustrated in Fig. 1. It consists of a single-mode laser with a center wavelength of $532 \mathrm{~nm}$ and an output power of $125 \mathrm{~mW}$ in combination with a rotating diffuser (600 GRIT polish; diffusion angle $9^{\circ}$ ). After this rotating diffuser, a solid rectangular glass light pipe is placed. The purpose of this light pipe is to homogenize the incoming light through the many internal reflections of the light beam. The light pipe has an entrance and exit facet of $8 \times 15 \mathrm{~mm}^{2}$ and a length of $13 \mathrm{~cm}$.

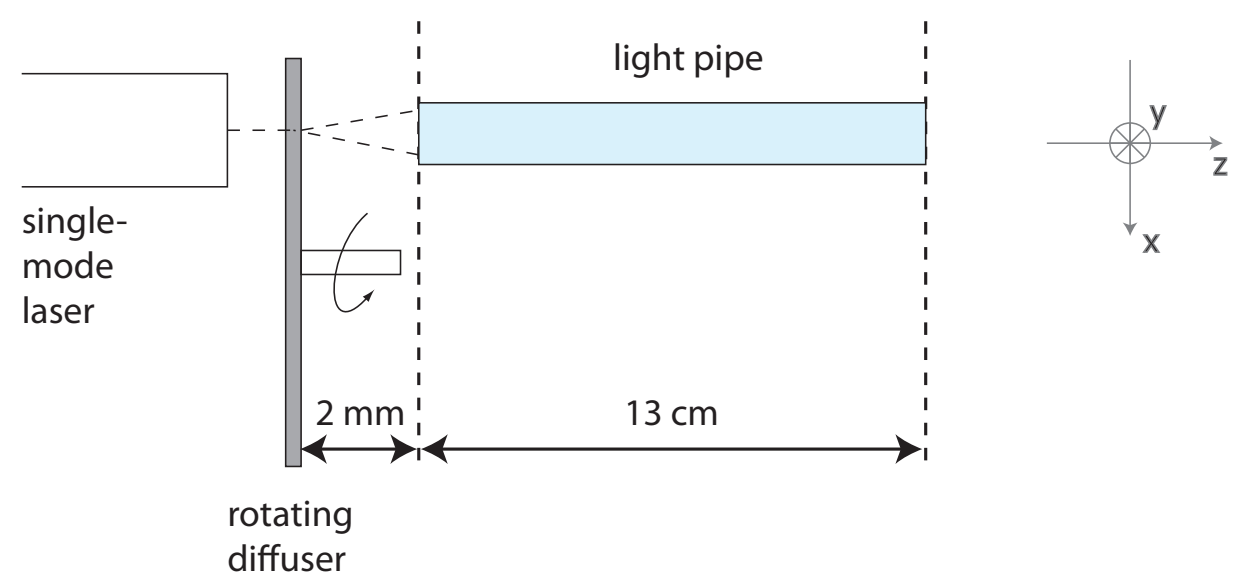

Figure 1. The investigated setup consists of a single-mode laser in combination with a rotating diffuser, closely followed by a solid rectangular glass light pipe with a length of $13 \mathrm{~cm}$. We also denote in the figure the axes orientation used in the modelling.

\subsection{Source model}

An incoherent superposition of identical, but spatially shifted coherent elementary fields are used to simulate the propagation of partially coherent light. ${ }^{9}$ The advantage of this approach is that the elementary fields are of identical functional form, thus only a single two-dimensional (2D) integration is necessary for their free-space propagation. The far-field radiant intensity pattern of the source determines the shape of the elementary field. 
Using a single mode laser with a Gaussian cross-section in combination with a rotating diffuser gives rise to a radiant intensity pattern that is very close to Gaussian. As a result, the shape of the elementary fields is also a Gaussian. This type of source -a Gaussian single mode laser and a rotating diffuser- can be described as a Gaussian Schell-model beam where both the intensity distribution and the complex degree of spatial coherence is of a Gaussian form. ${ }^{14-16}$ Hence in the remainder of this article, we shall consider this class of partially coherent beams only.

Gaussian Schell-model sources can be described analytically in close analogy to coherent Gaussian beams. This class also includes certain quasihomogeneous sources -such as light emitting diodes- for which the radius of the effective coherence area is much smaller than the effective source area. Gaussian Schell-model sources are common in practice, e.g. in the case of various lamps, light-emitting diodes, multi-mode fibers and pulsed broad-area vertical-cavity surface-emitting lasers. ${ }^{17,18}$

Gaussian Schell-model sources consist of a set of uncorrelated, identical, fully coherent, transversely shifted elementary modes of finite extent (see Fig. 2). The elementary modes are Gaussian and the diameter of the modes $w$ is determined by the diffusion angle using near-field to far-field Gaussian distribution formulas

$$
w=2 \frac{\lambda}{\pi \theta_{\text {diff }}},
$$

where $\theta_{\text {diff }}$ is the diffusion half angle (at $1 / \mathrm{e}^{2}$ ) and $\lambda$ is the wavelength. In our current situation, the resulting elementary mode diameter is equal to $2.1 \mu \mathrm{m}$, corresponding to a diffusion angle of $9^{\circ}$. These elementary modes are weighted by a function determined by the cross-spectral density of the source. This weighting function can be visualized at the plane of the diffuser and is also a Gaussian profile.



Figure 2. Intensity distribution in the transverse direction of the elementary Gaussian modes constituting the total source field. The source model consists of a Gaussian weighted linear superposition of spatially shifted but identical, fully coherent elementary Gaussian-shaped modes.

Only paraxial light propagation is considered such that the light can be described using a scalar field $U .{ }^{11}$ Further reduction of the computation time for the simulations is obtained by approximating the field as a spatially separable field in $x$ and $y$ dimensions with $U(x, y, z)=U_{x}(x, z) U_{y}(y, z)$. Such an approximation should not reduce the accuracy of the results significantly, as the light pipe is of a rectangular shape. Combination of propagated fields $U_{x}$ and $U_{y}$ provides a $2 \mathrm{D}$ wavefront at the observation plane. Simulation of the complex wave front is implemented by dividing both the aperture and observation plane into gridded meshes. The Rayleigh-Sommerfeld propagation method is implemented in a fast Fourier transform (FFT) approach. ${ }^{19}$

\subsection{Simulation model of the light pipe}

Homogenization in projection applications is possible making use of a light pipe. Such a component is in essence a rectangular rod in which light is reflected multiple times as it propagates through. These internal reflections 
are represented in the top of Fig. 3. As the elementary modes representing the source are transversely shifted, each of them reflect differently, giving rise to an irregular -but homogenized-intensity pattern at the exit facet of the light pipe. In the bottom part of Fig. 3, the implementation of the light pipe in the simulations is explained. Every elementary mode is propagated in free-space through glass until the end of the light pipe. Obviously, the intensity pattern at the end is still Gaussian. However, the light does not have the ability to simply propagate in free space, as it gets reflected at the sides of the light pipe. Fresnel reflections occur on the inside of the light pipe and depending on the incoming angle, corresponding Fresnel coefficients are applied. Consider for instance in Fig. 3 the blue rays of one elementary mode. They do not arrive at the surfaces $S^{-1}$ and $S^{1}$ at the exit plane of the light pipe, but are reflected onto the exit facet of the light pipe $S^{0}$. This internal reflection is simulated by mapping/transforming the wave-field at the exit plane of the light pipe onto the exit facet of the light pipe. Therefore, the fields of the surfaces $S^{-1}$ and $S^{1}$, are mirrored and added -on an amplitude basis- onto the field at the exit facet $S^{0}$ of the light pipe. The fields arriving in their neighboring surfaces $S^{-2}$ and $S^{2}$, are mirrored twice and again added onto the exit facet of the light pipe. This transformation process is performed for every elementary mode and each elementary mode intensity pattern is added to obtain the final intensity distribution at the end of the light pipe. The resulting intensity distribution after this mapping is exactly the same as the one shown in the top of the figure.

This simulation method is time-efficient is due to the following reasons:

- the field can be simulated using a 1D scalar field

- the light pipe transformation is obtained using a simple mapping algorithm

- the elementary modes are used to effectively describe the partially coherent field

This simulation model is not restricted to rectangular light pipes, but can also be extended toward tapered or more complex ones. The only alteration to the model should be to accurately describe the mapping transformation of the light pipe. As a result, the efficiency of the simulation is expected to remain high.
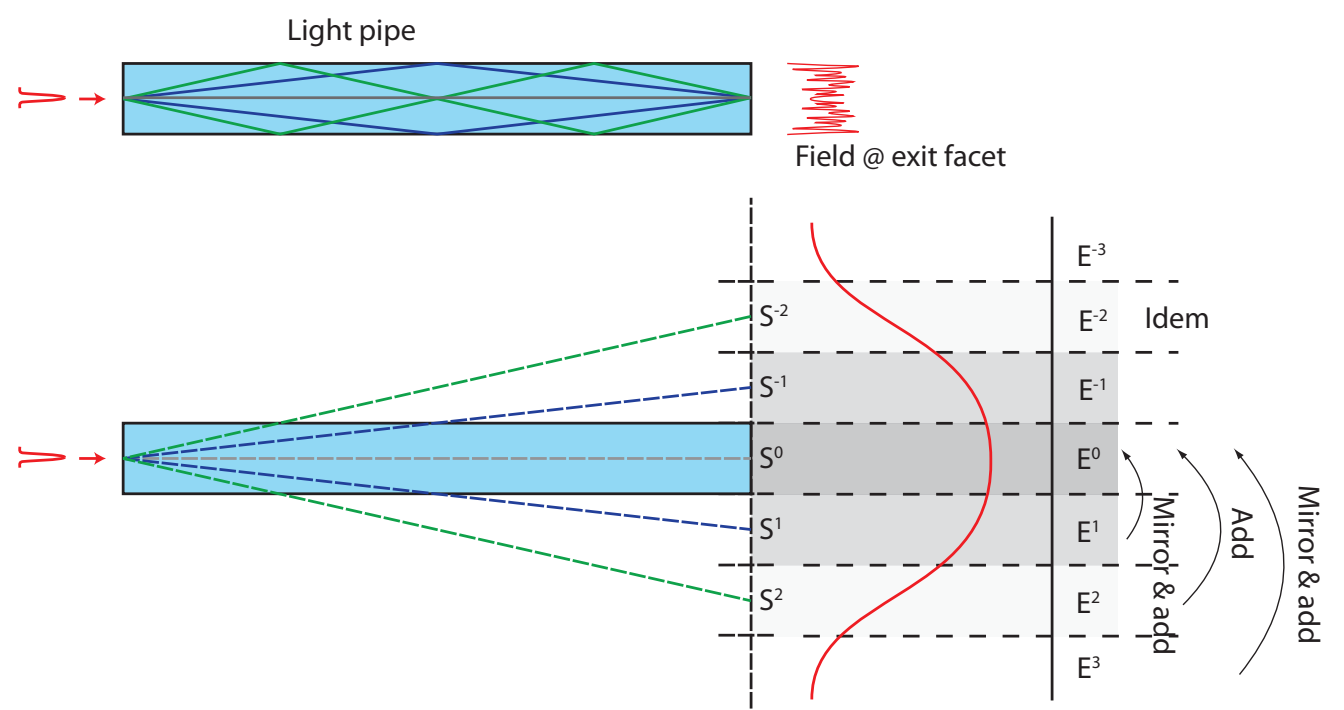

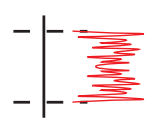

Field after mapping transformation

Figure 3. Top: propagation of rays from an elementary mode in the light pipe. Bottom: equivalent propagation of the same elementary mode through free-space propagation in glass and afterwards transforming/mapping the field to incorporate the internal reflections. 


\subsection{Calculation of the complex degree of coherence}

The coherence properties of the light can be investigated by calculating the complex degree of spatial coherence. In the case of partially coherent light consisting of a superposition of elementary modes, the (sampled) mutual coherence function at two pixels $l$ and $m$ in the detector plane takes on the form

$$
\Gamma_{\operatorname{lm}}=\sum_{\text {elem.modes }} U^{l} U^{m *},
$$

where $U^{l}$ is the field of one elementary mode at the plane of the detector at pixel $l$. Consequently, the complex degree of coherence is given by

$$
\gamma_{\mathrm{lm}}=\frac{\Gamma_{\mathrm{lm}}}{\sqrt{\Gamma_{\mathrm{ll}} \Gamma_{\mathrm{mm}}}} .
$$

Essentially, the field at pixel $l$ is multiplied with the conjugate of the same field at pixel $m$. This is performed for every elementary beam and then the results are summed. In the remainder of this paper, we visualize the coherence properties of a field by taking the magnitude of the complex degree of coherence between the center point of the field and other points along its transverse extent. The resulting graph thus describes the spatial coherence properties of the light as a function of the transverse extent.

\section{EXPERIMENTAL EVALUATION OF THE SIMULATION MODEL}

The spatial coherence properties at the end of the light pipe are measured using a reversing wavefront Michelson interferometer (RWI), ${ }^{20-22}$ as illustrated in Fig. 4. The exit facet of the light pipe is imaged onto the CCD with a magnification of 31, using an aspherical lens with a focal length of $20 \mathrm{~mm}$. The light beam is split by a $50 / 50$ beam splitter towards a gold coated mirror and a retro reflector prism. The former simply reflects the light whereas the latter additionally rotates the light $180^{\circ}$ in the plane transverse to the optical path. Both beams are directed toward the same point on the CCD. Maximal fringe visibility is obtained by translating the gold coated mirror in the longitudinal direction. This visibility measurement visualizes the magnitude of the complex degree of spatial coherence.

Interference is measured between the field at $\mathbf{r}_{1}=\left(x_{1}, y_{1}\right)$ and $\mathbf{r}_{2}=\left(-x_{1},-y_{1}\right)$, where the position $(0,0)$ corresponds to the center of the interferogram. Therefore, radially symmetric points with respect to the interferogram's center are compared. If the field in $\mathbf{r}_{\mathbf{1}}$ is coherent with the field in $\mathbf{r}_{\mathbf{2}}$, interference fringes occur at those positions in the CCD plane that correspond to $\mathbf{r}_{\mathbf{1}}$ and $\mathbf{r}_{\mathbf{2}}$.

The magnitude of the complex degree of coherence is calculated via ${ }^{21}$

$$
|\gamma|=\frac{\sqrt{\left[I_{t}^{0}-I_{0}^{H}-I_{0}^{V}\right]^{2}+\left[I_{t}^{\lambda / 4}-I_{0}^{H}-I_{0}^{V}\right]^{2}}}{2 \sqrt{I_{0}^{H} I_{0}^{V}}},
$$

where $I_{t}^{0}$ is the interference pattern, $I_{t}^{\lambda / 4}$ is the interference pattern obtained after shifting one of the interferometer arms by a quarter of a wavelength, $I_{0}^{H}$ is the intensity distribution when the light from the retro-reflector is blocked and $I_{0}^{V}$ is the intensity distribution when the light from the mirror is blocked.

\section{COMPARISON OF THE SIMULATION RESULTS WITH THE EXPERIMENTS}

The usage of a light pipe does change the partial coherence properties of the light. In the following section, two different situations are compared and discussed, namely free space propagation in air over a path length equal to that of the light pipe, and propagation through the light pipe.

The elementary modes representing the partially coherent field at the diffuser plane are Gaussian beams with a diameter of $2.1 \mathrm{\mu m}$. This size was verified using the interferometer setup, as referred to in Fig. 4 , where the diffuser plane is imaged onto the detector. The extent of the source beam $W$ was measured to be $780 \mu \mathrm{m}$. 


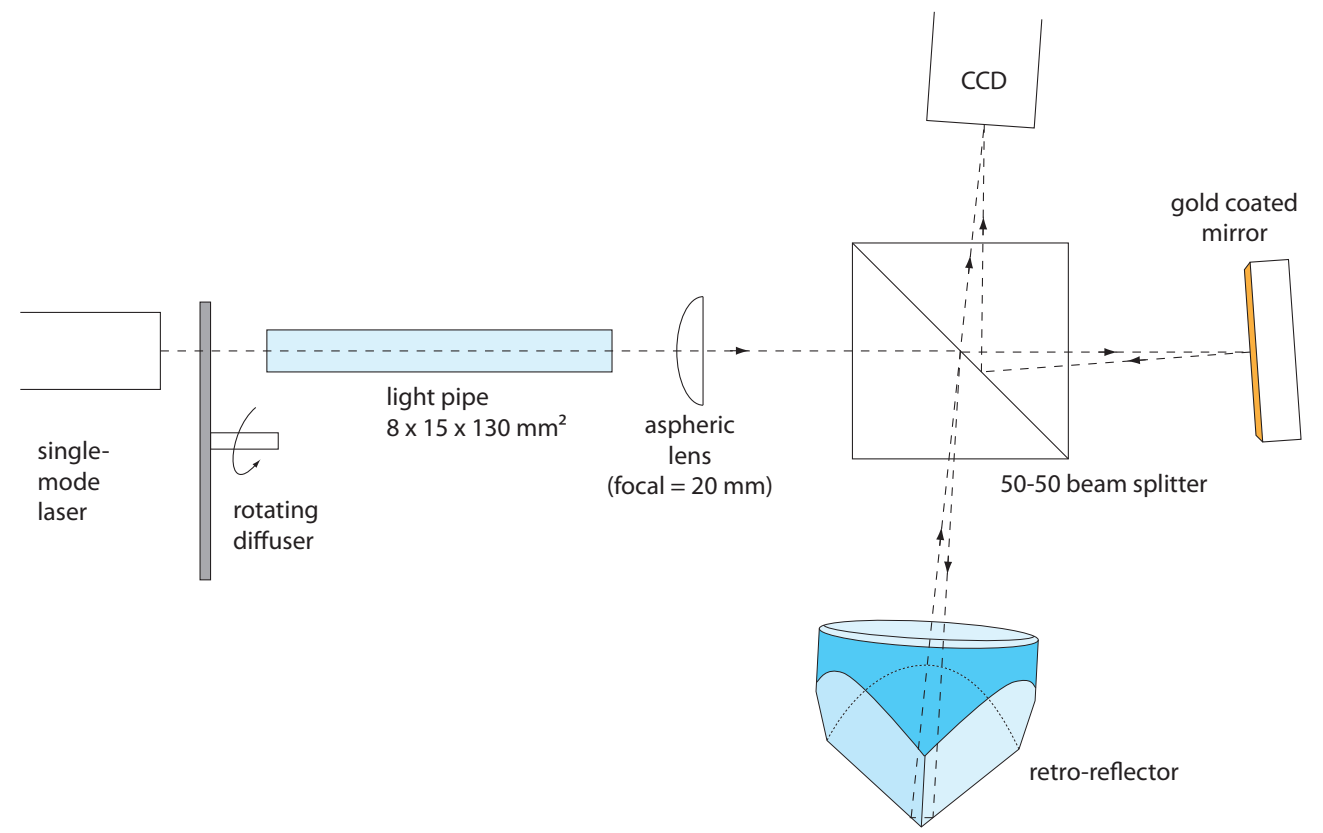

Figure 4. Setup of a reversing wavefront Michelson interferometer used to measure the spatial coherence area at the end of the light pipe.

\subsection{Free space propagation}

The influence of a light pipe on the partially coherent light beam is investigated by comparing propagation through the light pipe with free space propagation over an equivalent distance in air, namely $L_{\mathrm{LP}} / n=$ $13 \mathrm{~cm} / 1.5=8.67 \mathrm{~cm}$.

The outcomes of the free space propagation are depicted in Fig. 5. In Fig. 5(a), the simulation result of the degree of spatial coherence is depicted as a function of the transverse direction. The blue marks represent the simulation model and the red dashed curve represents the theoretical value of the magnitude of the complex degree of coherence for a Gaussian Schell-model beam propagated over the same distance. ${ }^{23}$ The simulation model and the theoretical function correspond very well and the spatial coherence diameter can be calculated. It is defined as the distance over which the degree of coherence has dropped to the value $1 / e^{2}$. In this situation, the coherence diameter is $75 \mu \mathrm{m}$. As a result, points in the observation plane that are separated by a distance larger than $75 \mu \mathrm{m}$ are considered uncorrelated.

The complex degree of coherence after free-space propagation is also measured using the interferometer setup depicted in Fig. 4. Its result is depicted in Fig. 5(b). Here, also a coherence area diameter is measured by taking into account the magnification and pixel size of the CCD. The overlapping weight function is indeed Gaussian. Note that the interferometer setup uses a retro-reflector and thus it measures the correlation $\gamma\left[-\mathbf{r}_{1}, \mathbf{r}_{1}, 0\right]$ between points that are radially symmetric with respect to the correlation measurement's center (which is the center of Fig. 5(b)). The diameter of the interference pattern in Fig. 5(b) thus corresponds to the radius of the coherence area in Fig. 5(a).

The small variation from the Gaussian shape in Fig. 5(b) are the result of the fact that image $I_{t}^{\lambda / 4}$ was not measured exactly at a phase shift of $90^{\circ}$ with respect to $I_{t}^{0}$ as was described in Eq. (4). Additionally, the "lines" at the angles $\pm 45^{\circ}$ are the result of using a retro-reflector. The light from the beam splitter is reflected towards this retro-reflector and is incident very close to its center. As a result, some distortion arises. 


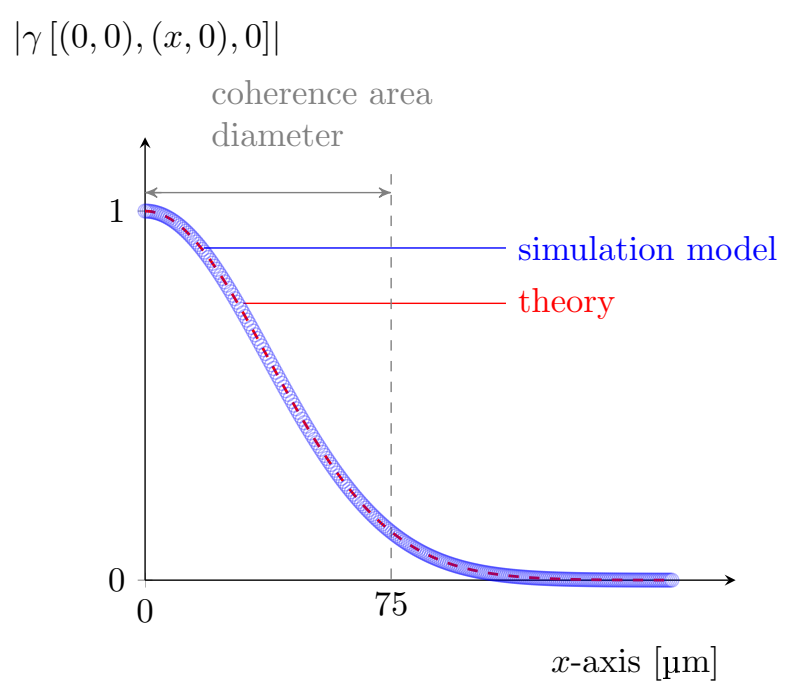

(a) Complex degree of coherence $|\gamma[(0,0),(x, 0), 0]|$ between the beam's center position and a transverse point at a distance $x$ from the center (for a single mode laser beam passing through a rotating diffuser) for free-space propagation over a distance of $8.67 \mathrm{~cm}$ after the diffuser. Blue marks: simulation, green curve: analytical value. The profile of the degree of coherence is Gaussian and the coherence diameter is $75 \mu \mathrm{m}$.

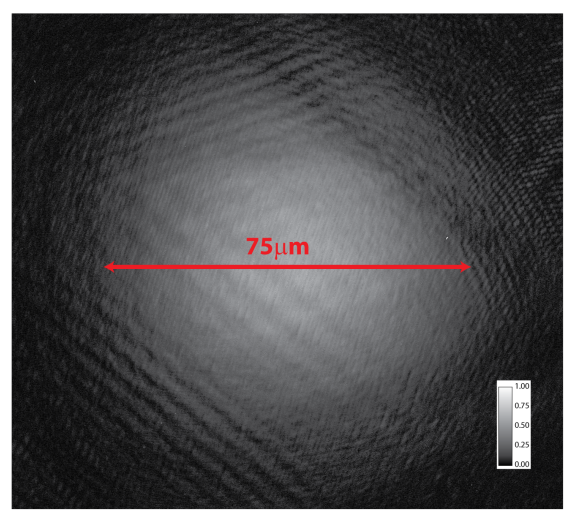

(b) Intensity map of the degree of coherence $|\gamma[(-x,-y),(+x,+y), 0]|$ measured at the CCD of the interferometer setup for free-space propagation over $8.67 \mathrm{~cm}$. The coherence diameter is found to be $75 \mu \mathrm{m}$.

Figure 5. Free space propagation results.

\subsection{Influence of the light pipe on the complex degree of coherence}

Usage of a light pipe has an influence on the partially coherent properties of light. This can be seen in the simulation result, depicted in Fig. 6, where the degree of spatial coherence is measured for the situation described in Fig. 1. The blue curve represents the degree of coherence obtained from the simulations of the light pipe. The red dashed curve represents the degree of coherence for free-space propagation over the equivalent length (Section 4.1). In comparison with the degree of coherence for the FSP (Fig. 5), here it no longer exhibits a Gaussian profile, but rather a profile of equidistant peaks with a slowly-varying Gaussian envelope. These peaks are the result of internal reflections in the light pipe. The width of the envelope function is the same as that of the free-space propagation, namely $75 \mu \mathrm{m}$.

This result is also experimentally verified using the RWI where the degree of coherence at the end of the light pipe is visualized on the CCD camera and depicted in Fig. 7(a). The same -spiked- intensity profile pattern underneath a Gaussian weighting function with a of $75 \mu \mathrm{m}$ is found. The fact that both simulation and experiments correspond well shows the validity of the model described in Section 2. From the simulations, we can obtain more insight in the effect of the light pipe. Because the source is quasi-homogeneous, the farfield coherence function $\mathrm{FF}_{\mathrm{coh}}$ is defined by the Fourier transform $\mathcal{F}$ of the near-field intensity. The near field intensity distribution at the rotating diffuser $\mathrm{NF}_{\text {int }}$ can be described as the convolution between a Gaussian profile (originating from the Gaussian single-mode beam impinging at the diffuser) and a spatial comb (as a result of the internal reflection inside the light pipe), i.e. in $1 \mathrm{D}$ this can be written as

$$
\begin{aligned}
& \mathrm{NF}_{\text {int }} \propto \exp \left(-a x^{2}\right) *\left[\sum_{k} \delta(x-k D)\right] \\
& \mathrm{FF}_{\text {coh }} \propto \mathcal{F}\left\{\mathrm{NF}_{\text {int }}\right\},
\end{aligned}
$$

where $\exp \left(-a x^{2}\right)$ is a Gaussian function with a width equal to the width of the single-mode beam impinging on the diffuser, $D$ is the transverse size of the light pipe and $x$ is the transverse coordinate in the near field. As a 




Figure 6. The degree of coherence for the light of our partially coherent source that has propagated through the $13 \mathrm{~cm}$ long light pipe. It exhibits a spiked profile with a slowly-varying envelope of width $75 \mu \mathrm{m}$.

result, the profile of the complex degree of coherence in Fig 6 is the product between a Gaussian envelope and a comb,

$$
\mathrm{FF}_{\text {coh }} \propto \mathcal{F}\left\{\exp \left(-a x^{2}\right)\right\} \cdot\left[\frac{1}{D} \sum_{k} \delta\left(\xi-\frac{k}{D}\right)\right],
$$

where $\xi$ is the spatial frequency in the far-field. Consequently, the far-field grid spacing $x_{\text {grid }}$ is given by

$$
x_{\text {grid }}=\frac{1}{2} \frac{\lambda z}{D}
$$

where $\lambda$ is the wavelength, $z$ is the longitudinal propagation distance from near field to the exit facet of the light pipe. Therefore, if the width of the light pipe is reduced, the distance between the peaks in Fig. 6 will increase. Note that the factor $1 / 2$ is the result of the fact that the reversing wavefront interferometer measures the coherence of radially symmetric points (cfr. Section 3 ).

The different period of the 2D interference pattern in Fig. 7(a) in the vertical and horizontal direction are due to the rectangular transverse shape of the light pipe. Recall that the dimensions of the light pipe entrance and exit facets are $8 \times 15 \mathrm{~mm}^{2}$, i.e. the width of the light pipe is approximately half of the height. This is also experimentally verified by the authors using the period of the far-field interference pattern in Fig. 7.

A cross-section profile of the degree of coherence is taken in the horizontal direction and is depicted in Fig. 7(b). One has to remark that in theory, the degree of coherence should reach a zero value between two consecutive peaks of the grid pattern for a source with a low degree of coherence. This is not obtained in practice because in order to calculate Fig. 7(a), using Eq. (4), two interference patterns are measured for which the only difference between them is a lateral translation in one of the interferometer arms with a distance of $\lambda / 4$. The measurements are not taken instantly, so some fluctuations arise resulting in a broadening of the grid pattern. Additionally, the grid-like pattern will be somewhat blurred due to the finite size of the CCD pixels and due to optical imperfections in the interferometer. The sum of these external factors results in a fringe visibility that does not reach zero for a low degree of coherence.

From the previous analysis, it is clear that the light pipe influences the coherence properties of the light and we are able to model this behavior accurately with a fast and efficient simulation model. The influence of the 




(a) Intensity map of the magnitude of the degree of coherence, exhibiting an interference pattern with a slowly varying envelope of width $75 \mu \mathrm{m}$. cross-section

$|\gamma[(-x, 0),(+x, 0), 0]|$

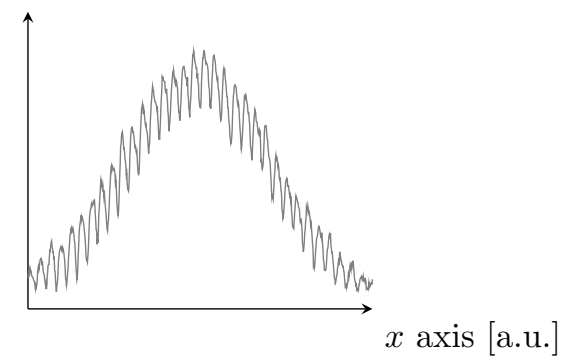

(b) The cross-section of (a) at the center of the degree of coherence pattern.

Figure 7. The magnitude of the degree of coherence at the exit facet of the light pipe, measured at the detector of the interferometer setup (a) and a cross-section taken at the center (b).

light pipe on the degree of coherence is visible in the fact that it transforms the free space propagation degree of coherence from a Gaussian into a rather spiked profile underneath the same Gaussian distribution (see Fig. 6). This implies that specific neighboring points inside the original coherence area appear to have no correlation with each other. Thus, they are not able to interfere. Owing to the rather complicated propagation of light through the light pipe, the light at specific neighboring points at the exit plane of the light pipe originate from distant points at the input plane. Since the fields at these distant points are only weakly correlated, the same can also be said about neighboring points at the exit plane. For a fully coherent laser beam, neighboring points at the exit plane are still fully correlated and the grid pattern will not appear in the complex degree of coherence at the exit plane of the light pipe.

\section{SUMMARY \& CONCLUSION}

The coherence properties of partially coherent light are influenced by the usage of a light pipe in comparison with free space propagation. This is confirmed using both simulations and experiments. A fast and efficient simulation model is designed for the propagation of partially coherent light, in which the light pipe is incorporated as a mapping transformation of the wave field. The coherence properties in both situations are clearly different and the consequences are discussed. As speckle is a coherence effect, observed as a granular pattern on the projection screen, we will as a future task employ our simulation model to investigate the effect of micro-structured projection screens to reduce the speckle. ${ }^{4,24}$ We can conclude that the usage of a light pipe does change the coherence properties of the light. These outcomes have also been experimentally verified making use of a reversing wavefront Michelson interferometer. A light pipe alters the degree of coherence of the partially coherent light in such a way that certain neighboring points inside the original coherence area are no longer correlated with one another. This implies that the field at these points do not interfere anymore and could reduce the speckle appearance on the screen. This aspect will be investigated in future work together with the interaction of partially coherent light with micro-optical components. The simulation model is currently implemented as a 1D propagation method, but this does not yet pose any shortcomings. Furthermore, this investigation method can also be applied to homogenizing light pipes that have other, more complex shapes. 


\section{Acknowledgments}

The authors would like to acknowledge financial support from the Agency for Innovation by Science and Technology in Flanders (IWT), the Fund for Scientific Research (FWO), the Industrial Research Funding (IOF), Methusalem VUB-GOA, IAP BELSPO VI-10 under grant IAP P7-35 "photonics@be", the OZR of the Vrije Universiteit Brussel, and the Academy of Finland (grant 118951).

\section{REFERENCES}

[1] Chellappan, K., Erden, E., and Urey, H., "Laser-based displays: a review," Appl. Opt. 49(25), 79-98 (2010).

[2] Hecht, J., "A short history of laser development," Appl. Opt. 49(25), 99-122 (2010).

[3] Weichmann, U., Bellancourt, A., Mackens, U., and Moench, H., "Solid-state lasers for projection," Journal of the Society for Information Display 18, 813 (2010).

[4] Goodman, J. W., [Speckle phenomena in optics: theory and applications], Roberts and Company, Englewood (2007).

[5] Roelandt, S., Meuret, Y., Craggs, G., Verschaffelt, G., Janssens, P., and Thienpont, H., "Standardized speckle measurement method matched to human speckle perception in laser projection systems," Opt. Express 20, 8770-8783 (Apr. 2012).

[6] Lowenthal, S. and Joyeux, D., "Speckle removal by a slowly moving diffuser associated with a motionless diffuser," JOSA 61(7), 847-851 (1971).

[7] Mandel, L. and Wolf, E., [Optical Coherence and Quantum Optics], Cambridge University Press, 1 ed. (Sept. 1995).

[8] Gori, F. and Palma, C., "Partially coherent sources which give rise to highly directional light beams," Opt. Commun. 27, 185-188 (Nov. 1978).

[9] Vahimaa, P. and Turunen, J., "Finite-elementary-source model for partially coherent radiation," Opt. Express 14, 1376-1381 (Feb. 2006).

[10] Peeters, M., Verschaffelt, G., Speybrouck, J., Thienpont, H., Danckaert, J., Turunen, J., and Vahimaa, P., "Propagation of spatially partially coherent emission from a vertical-cavity surface-emitting laser," Opt. Lett. 31, 1178-1180 (May 2006).

[11] Roelandt, S., Tervo, J., Meuret, Y., Verschaffelt, G., and Thienpont, H., "Propagation of partially coherent light through a light pipe," Opt. Express 21(14), 17007-17019 (2013).

[12] Wyrowski, F., "Field tracing for unified optical modeling," in [Frontiers in Optics], FW4A-1, Optical Society of America (2012).

[13] Riechert, F., Speckle Reduction in Projection Systems, PhD thesis, Karlsruhe Institute of Technology (KIT) (2009).

[14] De Santis, P., Gori, F., Guattari, G., and Palma, C., "An example of a collett-wolf source," Opt. Commun. 29(3), 256-260 (1979).

[15] Farina, J., Narducci, L., and Collett, E., "Generation of highly directional beams from a globally incoherent source," Opt. Commun. 32(2), 203-208 (1980).

[16] He, Q., Turunen, J., and Friberg, A. T., "Propagation and imaging experiments with gaussian schell-model beams," Opt. Commun. 67(4), 245-250 (1988).

[17] Peeters, M., Verschaffelt, G., Thienpont, H., Mandre, S., Fischer, I., and Grabherr, M., "Spatial decoherence of pulsed broad-area vertical-cavity surface-emitting lasers," Opt. Express 13(23), 9337-9345 (2005).

[18] Mandre, S. K., Elsäßer, W., Fischer, I., Peeters, M., and Verschaffelt, G., "Evolution from modal to spatially incoherent emission of a broad-area vcsel," Opt. Express 16(7), 4452-4464 (2008).

[19] Shen, F. and Wang, A., "Fast-fourier-transform based numerical integration method for the rayleighsommerfeld diffraction formula," Appl. Opt. 45(6), 1102-1110 (2006).

[20] Santarsiero, M. and Borghi, R., "Measuring spatial coherence by using a reversed-wavefront young interferometer," Opt. Lett. 31(7), 861-863 (2006).

[21] Imai, M., Ohtsuka, Y., and Satoh, S., "Spatial coherence analysis of light propagation in optical fibers by interferometric methods," JOSA A 3(7), 1059-1064 (1986). 
[22] Verschaffelt, G., Craggs, G., Peeters, M., Mandre, S. K., Thienpont, H., and Fischer, I., "Spatially resolved characterization of the coherence area in the incoherent emission regime of a broad-area vertical-cavity surface-emitting laser," IEEE Journal of Quantum Electronics 45(3), 249-255 (2009).

[23] Born, M. and Wolf, E., [Principles of optics: electromagnetic theory of propagation, interference and diffraction of light], Cambridge University Press (1999).

[24] Riechert, F., Craggs, G., Meuret, Y., Van Giel, B., Thienpont, H., Lemmer, U., and Verschaffelt, G., "Lowspeckle laser projection with a broad-area vertical-cavity surface-emitting laser in the nonmodal emission regime," Appl. Opt. 48, 792-798 (Feb. 2009). 